\title{
Numerical Simulation Study on Flow Characteristics of Supercritical Carbon Dioxide in a New Cleaning Chamber
}

\author{
Guangya Jin ${ }^{\mathrm{a}}$, Ya Wang ${ }^{\mathrm{b}}$, Jiapeng Wang ${ }^{\mathrm{c}}$, Songyang Zhu ${ }^{\mathrm{d}}$ \\ Department of Power Engineering, School of Energy, Power \& Mechanical Engineering, North China \\ Electric Power University, Baoding 071003, China

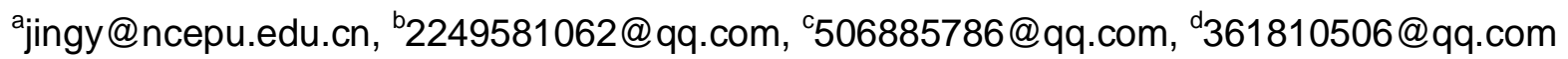

\begin{abstract}
Keywords: Supercritical carbon dioxide, cleaning chamber, flow characteristics, wall shear stress Abstract: Large amounts of organic solvents and pure water have been consumed in conventional semiconductor cleaning technology. And low dielectric constant (Low-K) material may be destroyed easily during the cleaning process, which will also cause structural collapse of the material. All these shortcomings can be rid by cleaning technique using supercritical carbon dioxide $\left(\mathrm{SC}-\mathrm{CO}_{2}\right)$ as a medium. As an environmental friendly solvent, $\mathrm{SC}-\mathrm{CO}_{2}$ has been applied in wafer cleaning field for some unique advantages such as high solvency, high diffusivity, low viscosity, low surface tension and other characteristics. In this study, a new cleaning chamber using $\mathrm{SC}-\mathrm{CO}_{2}$ is designed and a simplified model is established by CFD software. On this basis, the flow field characteristics of $\mathrm{SC}-\mathrm{CO}_{2}$ in the chamber are simulated, and the corresponding wall shear stress distribution is obtained by altering the rotation speed of the inner cylinder and the outlet pressure of multi-directional nozzles. The simulation results aim to offer ideas for expanding applications of $\mathrm{SC}-\mathrm{CO}_{2}$ as a green solvent.
\end{abstract}

\section{Introduction}

In recent years, with the rapid development of microelectronics and other high-tech industries, semiconductor devices and metal interconnects inside the components become more and smaller. Besides, low dielectric constant materials and high aspect ratio features are applied more and more popular. As is well known, silicon wafer is the most widely used substrate material in semiconductor and VLSI industry, and during the manufacturing process, in addition to the exclusion of outside sources, the wafer surface cleaning is required almost every step in order to effectively remove variety adhered impurities on silicon surface, trenches and blind holes. Currently, the semiconductor cleaning technology is still based on the traditional RCA cleaning method, which has occupied a leading position due to low cost, safety, impurities and substrate selectivity, contaminant removal rate and other advantages [1]. In recent years, the traditional cleaning technology has been developing rapidly, but the microelectronics and other high-tech cleaning industry still faces many problems. As the features shrink to nano-scale, the cleaning solution contained in the acid and alkali and strong oxidizing agents can cause surface microroughness and structural deformation, aqueous cleaning is unfeasible due to surface tension and capillary forces increase [2-4]. Nanoporous silica (NPS) is a hotspot in ultra-low dielectric constant material domain, but highly corrosive chemical cleaning will destroy the material, the capillary force of water can also cause structural collapse [5]; The traditional cleaning has consumed a great deal of pure water and organic solvent, which inevitably caused serious environmental pollution, physical hazards and water waste. Therefore, looking for environmental friendly solvent is a trend for microelectronics and other high-tech technology.

As a special solvent with high solvency, high diffusivity, low viscosity, low surface tension and other characteristics, supercritical fluid is a most potential environmental substitute for organic solvent and an aqueous solvent. Supercritical carbon dioxide $\left(\mathrm{SC}-\mathrm{CO}_{2}\right)$ is relatively easy to implement $\left(\mathrm{T}>31.19^{\circ} \mathrm{C}, \mathrm{P}>7.38 \mathrm{MPa}\right)$, and it is not only nontoxic, noncorrosive, inert, low cost and environmental friendly, but also has strong permeability, detergency and sterilization, therefore it is a complete "green solvents". In general, the solubility of supercritical fluid increased with increased density, which will increase with increased pressure at a constant temperature.

In practical applications, cleaning chamber as one of the main equipment in cleaning system, undertakes the important task of removing the impurities [6]. The effect of cleaning quality mainly 
depends on the design of the chamber. Therefore, the design and development of efficient cleaning chamber is of great significance. In this study, a new cleaning chamber using $\mathrm{SC}-\mathrm{CO}_{2}$ as cleaning medium is described, and a simplified model is established by CFD software. On this basis, the flow field characteristics of SC-CO $\mathrm{CO}_{2}$ in the chamber are simulated, and the corresponding wall shear stress distribution is obtained by altering the rotation speed of the chamber and multi-nozzle outlet pressure. The simulation results aim to offer ideas for expanding applications of $\mathrm{SC}-\mathrm{CO}_{2}$ as a green solvent.

\section{Numerical calculation model}

A simplified physical model of the new cleaning chamber is shown in Fig.1. Model of equal dimension is established by Gambit, the sizes of model are as follows, the diameter of inner cylinder is $600 \mathrm{~mm}$, the height is $300 \mathrm{~mm}$, the diameter of fluid inlet is $20 \mathrm{~mm}$, the diameter of outlet is $50 \mathrm{~mm}$, the diameter of multi-directional nozzles is $10 \mathrm{~mm}$, and wafer size is set to 8 inches. The wafer is vacuum adsorpted on the inner cylinder wall, without using any clamping device in order to avoid affecting the local cleaning. Multi-directional nozzles ensure that the supercritical fluid can be injected to the wafer surface, and dynamic pressure of fluid exerts wall shear stress which combine solvency to remove impurities. The inner cylinder of the chamber is rotated by the electromotor to increase disturbance for impurities dissolution and removal.

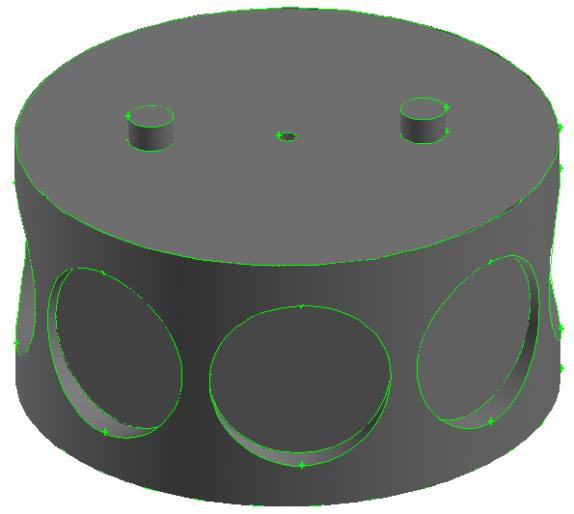

Fig.1 Physical model of the chamber

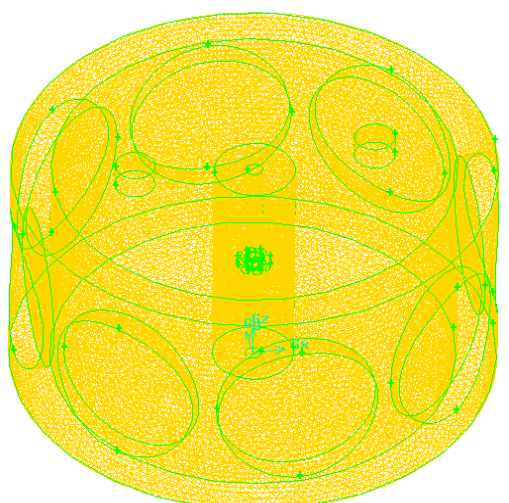

Fig.2 Schematic diagram of whole grid meshing

The real gas model in NIST database from CFD software to effectively simulate the actual cleaning process is adopted regarding various properties of $\mathrm{SC}-\mathrm{CO}_{2}$ [7]. The standard $\mathrm{k}-\varepsilon$ two equation turbulence model under high Reynolds number is chosen for establishing the mathematical model in this paper, and the second order upwind discretization model is added. Considering calculation accuracy in the jet process, the flow field grid near the nozzle is locally refined and number of mesh achieves 1921319. The schematic diagram of whole grid meshing is shown in Fig.2.

The inlet pressure is chosen as the chamber inlet boundary condition because it can be controlled easily by pumps and valves, and the inlet pressure is identified to range from $12 \mathrm{MPa}$ to $30 \mathrm{MPa}$. The mass outlet is chosen as the chamber outlet boundary condition because the outlet pressure and flow rate are hard to predict for the parameters changes with the changing pressure at the chamber inlet. For the solid surface boundary, the no-slip boundary method with the wall-function is employed in this study. To facilitate the calculation, the region of temperature space is from $306 \mathrm{~K}$ to $312 \mathrm{~K}$.

\section{Results and analysis}

When the inlet pressure is $15 \mathrm{MPa}$ and the rotation speed of inner cylinder is $300 \mathrm{rpm}$, the contours of velocity magnitude $(\mathrm{m} / \mathrm{s})(\mathrm{a})$ and streamtraces (b) in the chamber on the surface $\mathrm{Z}=0.15$ are shown in the Fig.3. According to results, it is shown that the velocity of $\mathrm{SC}-\mathrm{CO}_{2}$ decreased gradually and formed free diffusion from the nozzle to the silicon surface in the cleaning chamber, which is free submerged turbulent jet. The maximum velocity appeared in the multi-directional nozzle outlet; it is 
shown that the velocity of near wafer is inhomogeneous due to the effect of inner cylinder rotation, which will cause significant difference of the wall shear stress distribution on the wafer surface. It is shown in the streamtraces diagram postprocessing by Tecplot that a lot of the vortices are formed due to the combined effect of rotation and injection, and the position relative uniformly distributed.

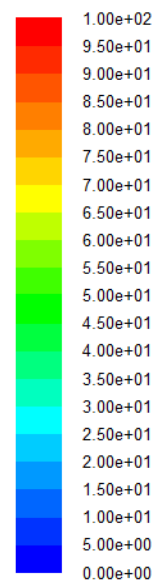

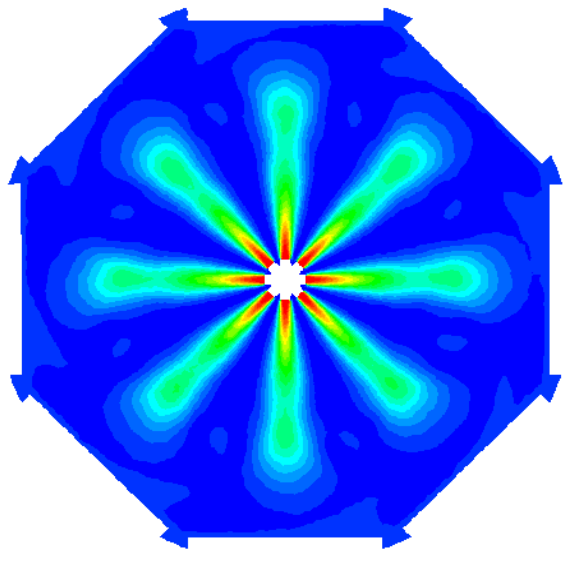

(a)

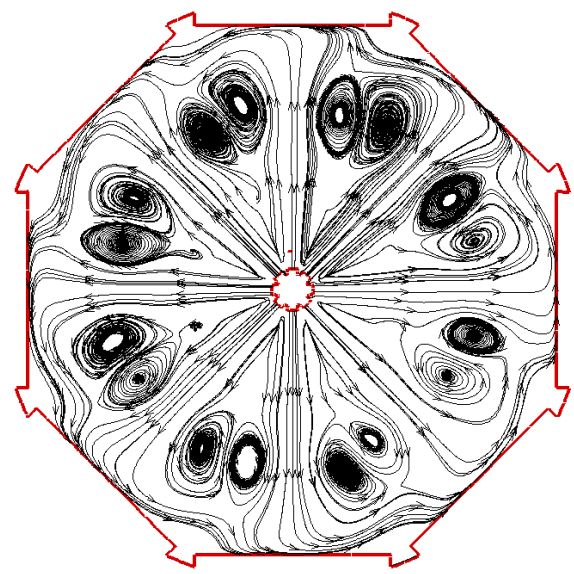

(b)

Fig.3 Contours of velocity magnitude (m/s) (a) and streamtraces (b) in the chamber on the surface $\mathrm{Z}=0.15$

The contours of velocity magnitude $(\mathrm{m} / \mathrm{s})$ (a) and streamtraces $(\mathrm{b})$ in the chamber on the surface $\mathrm{Y}$ $=0$ are shown in Fig.4. As seen from the contours of velocity magnitude, the velocity of $\mathrm{SC}-\mathrm{CO}_{2}$ gradually decreases from the nozzle to the wafer surface, which bring into correspondence with Fig.3 (a). In addition to the direct impact injection area, the larger disturbance area distributed on wafer surface, upper and lower sides of wafer and the cleaning chamber outlet. It is shown in the streamtraces diagram that the borderline is nozzle - wafer center line, and both upper and lower formed larger vortices respectively. The vortices are close to wafer surface, which will facilitate impurities removal from the wafer surface.

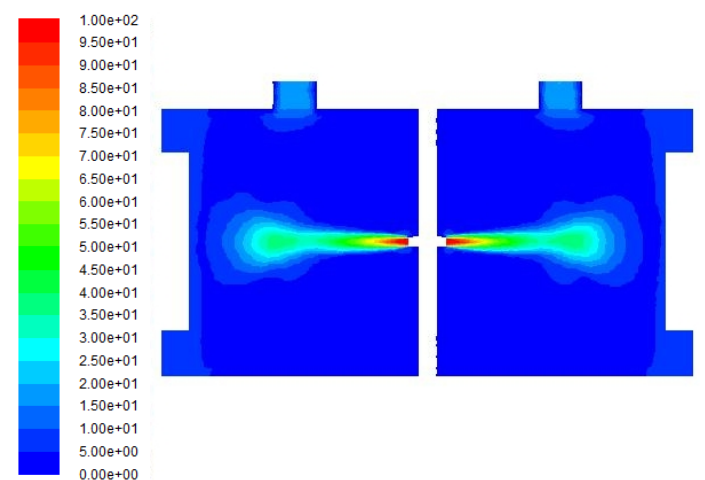

(a)

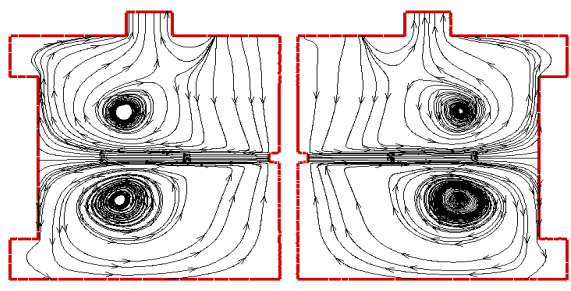

(b)

Fig.4 Contours of velocity magnitude (m/s) (a) and streamtraces (b) in the chamber on the surface $\mathrm{Y}=0$

When the inlet pressure is $15 \mathrm{MPa}$ and the rotation speed of inner cylinder is $1400 \mathrm{rpm}$, wall shear stress distribution of all wafers is shown in Fig.5. After comparison, wall shear stress of each wafer surface has very small difference; the maximum difference is only $1 \%$. Therefore, it is feasible to choose one of the wafers for analysis. In addition, the inner cylinder ran only in one direction of rotation, therefore wall shear stress of the wafer surface on both sides will appear very different phenomenon. In practical situation, the cleaning chamber can be controlled intermittently changing the direction of rotation, so that the wall shear stress of wafer surface can be uniform distributed. The 
value of wall shear stress may indirectly reflect the decontamination rate; although the relationship is not linear, qualitative analysis of cleaning effect is completely acceptable.

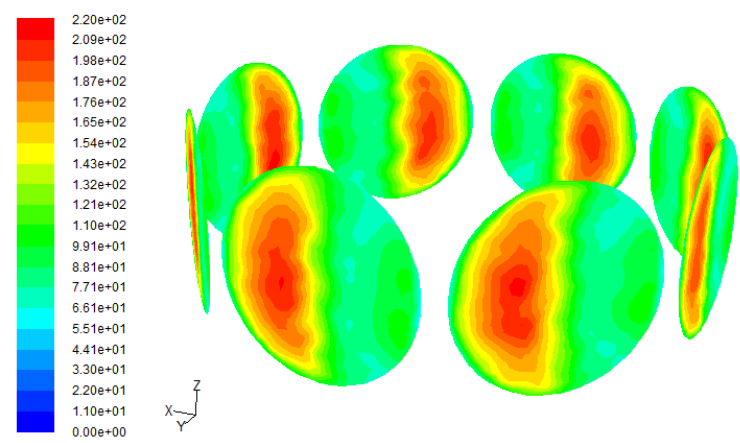

Fig. 5 Wall shear stress distribution of all wafer when the inlet pressure is $15 \mathrm{MPa}$ and rotation speed is $1400 \mathrm{rpm}$

When the inlet pressure is $15 \mathrm{MPa}$ and the rotation speed of inner cylinder is from 300 to $1400 \mathrm{rpm}$, wall shear stress comparison distribution of a single wafer is shown in Fig.6. As seen from the chart, the maximum wall shear stress increased with the increase of rotation speed, and the maximum value appeared in the center of the left area, and then decreased as semicircular from the center to the edge of the wafer. With the increase of rotation speed, the maximum value of the right wafer is located on the narrow area near the edge of the wafer, and the minimum value is always on the narrow region near the wafer center of the right side. If intermittently changing the direction of rotation, wall shear stress distribution of both sides in the region can be substantially uniform.

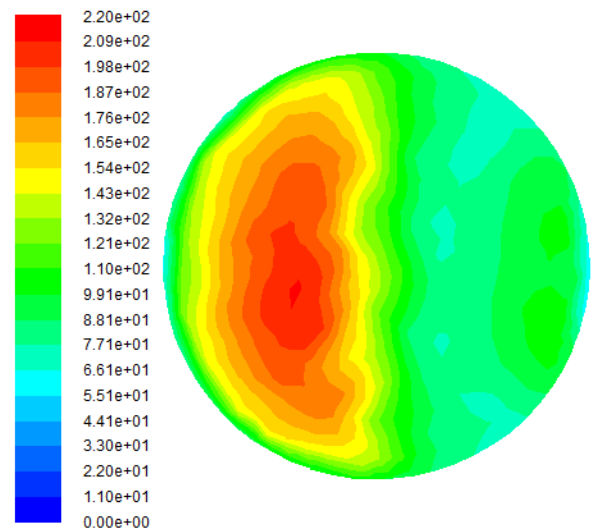

$(1400)$

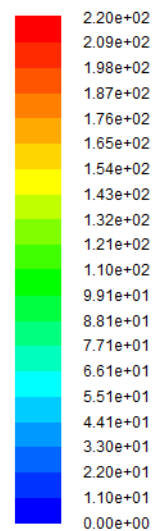

$1.10 \mathrm{e}+01$

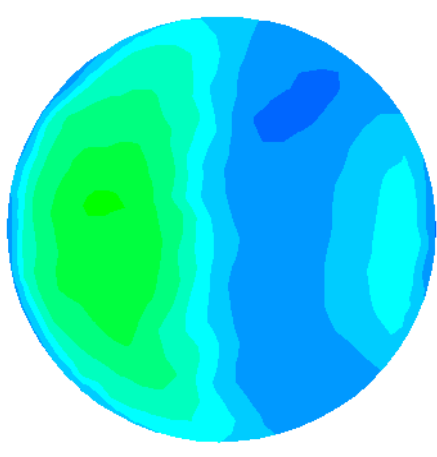

( 800$)$

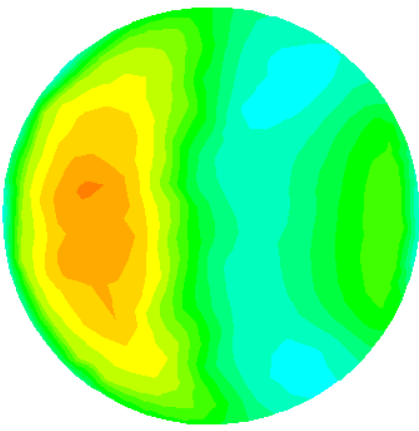

$(1200)$

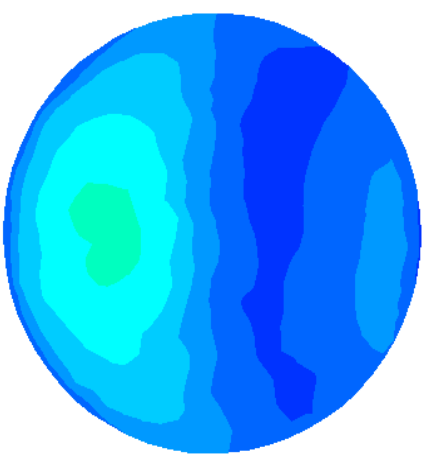

( 600 )

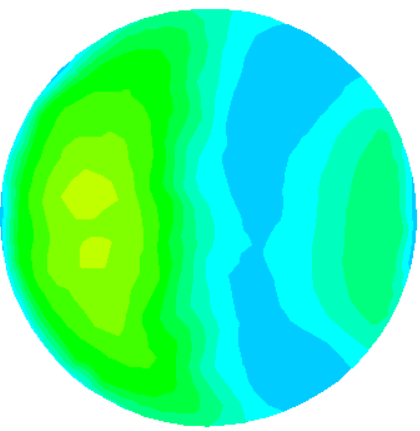

$(1000)$

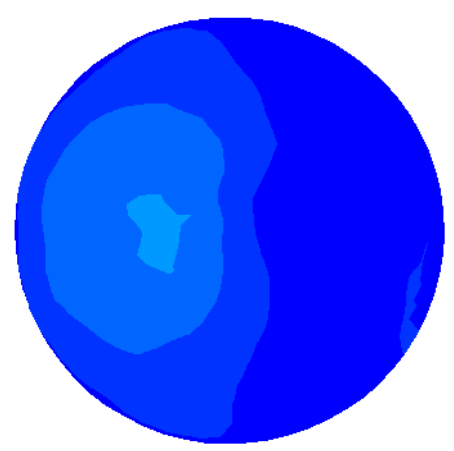

( 300 )

Fig.6 Wall shear stress comparison distribution of a single wafer when the inlet pressure is $15 \mathrm{MPa}$ and the rotation speed of inner cylinder is from 300 to $1400 \mathrm{rpm}$ 
When the rotation speed of inner cylinder is $1500 \mathrm{rpm}$ and the inlet pressure varies from 12 to $30 \mathrm{MPa}$, wall shear stress comparison distribution of a single wafer is shown in Fig.7. It is shown that wall shear stress distribution of a single wafer with the increase of inlet pressure maintained accordant with Fig.6. It is indicated that the wall shear stress can be increased by the increasing rotation speed of the inner cylinder to increase the disturbance, or the increase of the inlet pressure to increase hydrodynamic surface of the wafer.
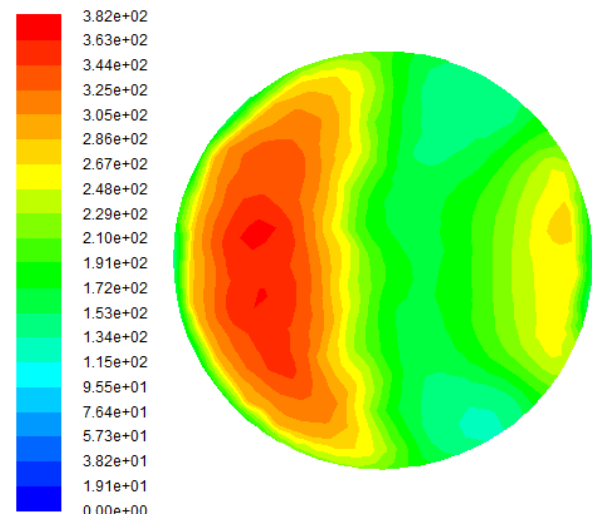

$(30 \mathrm{MPa})$

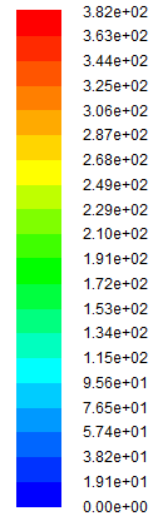

$1.91 \mathrm{e}+01$
$0.00 \mathrm{e}+00$

( 20MPa )

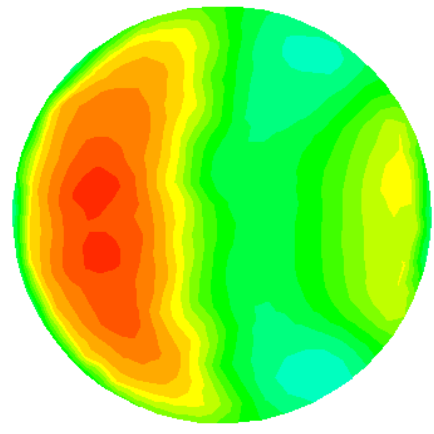

$(28 \mathrm{MPa})$

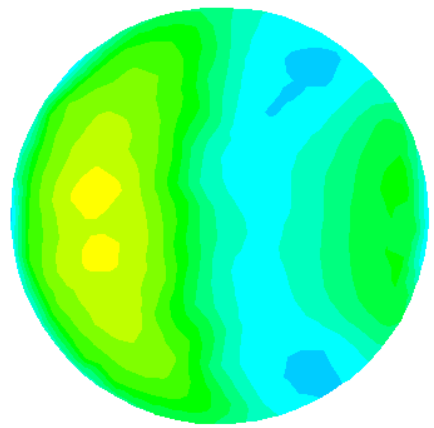

(16MPa)

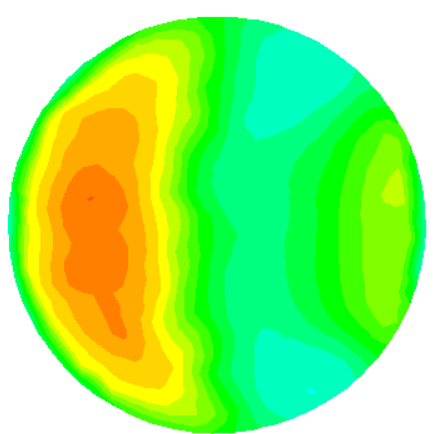

$(24 \mathrm{MPa})$

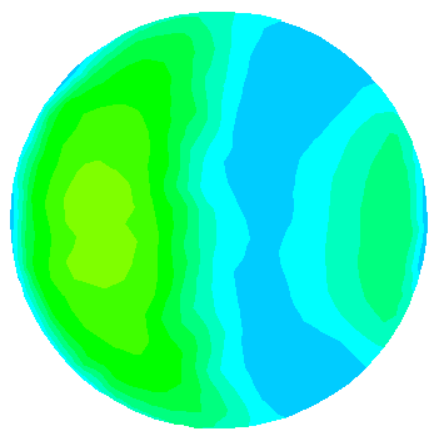

( $12 \mathrm{MPa})$

Fig.7 Wall shear stress comparison distribution of a single wafer when the rotation speed of inner cylinder is $1500 \mathrm{rpm}$ and the inlet pressure varies from 12 to $30 \mathrm{MPa}$

\section{Conclusions}

Conventional semiconductor cleaning technology has been unable to meet the developing requirements in new materials and micro-structure of the microelectronics industry, an environmental friendly solution is provided with supercritical fluid cleaning technology. The new cleaning chamber with supercritical carbon dioxide as cleaning solvent helps improving cleaning efficiency and better impurities removal by vacuum adsorption, multi-nozzle injection and inner rotation cylinder, as well as the perturbation simultaneously on multiple wafer cleaning.

Numerical simulation results of flow field characteristics in the cleaning chamber indicated that, free submerged turbulent jet of SC- $\mathrm{CO}_{2}$ is formed. Large areas were affected under the function of inner cylinder rotation, in addition to the direct impact injection area, the larger disturbance area distributed on wafer surface, upper and lower sides of wafer and the cleaning chamber outlet. The corresponding shear stress distribution of a single wafer surface was obtained at different rotation speed of inner cylinder and inlet pressure of the chamber, respectively. The simulation results indicated that cleaning effect can be enhanced by the increasing rotation speed of the inner cylinder to increase the disturbance, or the increase of the inlet pressure to increase hydrodynamic surface of the wafer. The simulation results aim to offer ideas for expanding applications of $\mathrm{SC}-\mathrm{CO}_{2}$ as a green solvent. 


\section{Acknowledgements}

This study is supported by the Fundamental Research Funds for the Central Universities (No.2014MS110) and Undergraduate Training Programs for Innovation and Entrepreneurship (No.201510054107).

\section{References}

[1] Liu W, Zhang B, Li Y, et al. Applied Surface Science, 2014, 292: 142-148.

[2] Hattori T. ECS Transactions, 2009, 25(5): 3-14.

[3] Zhang X G, Johnston K P. Chinese Science Bulletin, 2007, 52(1): 27-33.

[4] Ventosa C, Rébiscoul D, Perrut V, et al. Microelectronic Engineering, 2008, 85(7): 1629-1638.

[5] Keagy J A, Li Y, Green P F, et al. The Journal of supercritical fluids, 2007, 42(3): 398-409.

[6] Han T, Li B, Wang Q, et al. Microelectronic Engineering, 2012, 96: 1-5.

[7] DU Y, WANG R, NI H, et al. Journal of Hydrodynamics, Ser. B, 2013, 25(4): 528-534. 